\title{
AKAPs competing peptide HT31 disrupts the inhibitory effect of PKA on RhoA activity
}

\author{
YING WANG $^{1}$, YONGCHANG CHEN ${ }^{1}$, MIN CHEN $^{1}$ and WENRONG XU ${ }^{2}$ \\ ${ }^{1}$ School of Medicine, ${ }^{2}$ School of Medical Technology Jiangu University, P.R. China
}

Received April 4, 2006; Accepted June 29, 2006

\begin{abstract}
A-kinase can inhibit RhoA activation through phosphorylating $\operatorname{ser}^{188}$ of RhoA. AKAP is a novel protein that can target PKA to different subcellular compartment. Evidence has been presented that PKA anchorage by AKAP is important for the kinase to exert its function. This study analyzed the role of PKA anchorage in PKA-induced antagonism against RhoA activity and function. The cells transfected with pcDNA HT31wt/mut were treated with LPA and/or CPT-cAMP. The amount of GTP-RhoA and phosphorylation of RhoA was detected by Western blotting with specific antibodies. The formation of stress fiber was visualized under fluorescent microscope. The gene expression activity was analyzed by luciferase reporter gene assay. The motility and the anchorageindependent growth assays were carried out with stably transfected cells expressing the AKAP inhibitory peptide HT31. The results showed that HT31 not only blocked the PKA-induced phosphorylation of RhoA but also prevented the PKA-induced inhibition on RhoA activation. The disruption of PKA anchorage abolished its inhibition on the LPA-induced expression of reporter gene SRE-luciferase. The ability of PKA to antagonize the LPA-induced stress fiber formation was partly impaired upon the disruption of the PKA anchorage. The control of PKA on migration and the proliferation excited by LPA disappeared in stably transfected cells highly expressing HT31. The results revealed that PKA anchorage was necessary for the kinase to exert its inhibitory effect on RhoA activation and RhoA-dependent biological activities.
\end{abstract}

\section{Introduction}

RhoA protein is an important member of the Ras super family of low molecular weight $G$ proteins. It regulates the

Correspondence to: Dr Yongchang Chen, Jiangsu University, \#2 Dongwu Road, Zhenjiang City, Jiangsu Province 212001, P.R. China

e-mail:ycchen54@ujs.edu.cn

Key words: A-kinase, RhoA protein, AKAPs, peptide HT31 actin cytoskeleton, affects cell morphology and motility, and modulates gene expression, cell cycle progression, and cell survival (1-3). Although there are no reports on activating mutations of RhoA protein in human tumors, the protein is overexpressed in tumors (2). RhoA plays critical roles in cell transformation induced by oncogenic Ras, and constitutively active constructs of RhoA induce anchorage-independent growth and other features of the transformed phenotype $(2,4,5)$. The high incidence of overexpression of RhoA in human tumors suggests that the protein is important in the carcinogenic process, and therefore is a potential candidate for a therapeutic intervention.

cAMP-dependent kinase(A-kinase, PKA) was demonstrated to inhibit RhoA activation by phosphorylating RhoA on $\operatorname{ser}^{188}$ $(6,7)$. It has been reported that RhoA and PKA have antagonistic roles in regulating cellular morphology, motility, and anchorage-independent growth of cancer cells $(8,9)$. Studying the cross talk between PKA- and RhoA-mediated signal transductions will form the basis for new rational treatment strategies to control proliferation, invasion and metastasis of cancers.

A-kinase is a heterotetramer composed of two regulatory and two catalytic subunits. In mammalian cells, there are two types of PKA, type I and type II, which are distinguished by different regulatory subunits, RI and RII, as they share a common catalytic subunit (10). A-kinase is localized to subcellular organelles through association with A-kinase anchoring proteins (AKAPs), and this association is important for PKA action in specific cellular events (11). AKAPs bind the RII regulatory subunits of PKA through a common amphipathic helix motif and make the kinase close to its diverse substrates to ensure the specificity of cAMP-PKA pathway although the kinase has multiple substrates (12). HT31 is the peptide derived from human thyroid that can destroy the anchorage of A-kinase by competing with AKAPs, therefore is used to study the effect of PKA anchoring in multiple biochemical activities (13).

The importance of PKA anchorage in many biochemical activities, such as sperm motility and cardiac muscle contraction has been reported $(14,15)$, but the function of PKA anchorage in PKA inhibition on RhoA activity has not been completely addressed. Elucidating the roles of AKAPs in the crosstalk between PKA- and RhoA-mediated signal pathways will give us a better understanding of the mechanism, and possibly lead to therapy in clinic. 


\section{Materials and methods}

Cell lines. The human gastric epithelial cell line SGC-7901 and the transformed African green monkey kidney fibroblast cell line COS-7 were provided by the Institute of Cell Biology, Shanghai, P.R. China.

Reagents. Dulbecco's modified Eagle medium (DMEM) was from Gibco (Grand Island, NY); new-born calf serum (NBCS) was from Minhai Bio-engineering C. (Lanzhou, P.R. China); the antibody against RhoA was from Santa Cruz Biotechnology (Santa Cruz, CA); the antibody against RhoA phosphorylated at serine ${ }^{188}$ was from Calbiochem (San Diego, $\mathrm{CA}$ ); the horseradish peroxidase (HRP)-conjugated secondary antibody was from Jackson ImmunoResearch Laboratories (West Grove, PA); the cell transfection reagent, Lipofectamin 2000 was from Invitrogen (Carlsbad, CA); the cellular permeable cAMP analog 8-chlorophenylthio-cAMP (CPTcAMP) was from Calbiochem; lysophosphatidic acid (LPA) was from Sigma (St. Louis, MO); electrochemiluminescence (ECL) reagents were from Amersham Biosciences (Buckinghamshire, UK); all plasmid DNA constructs used in this experiment were kind gifts from Dr Renate Pilz (University of California, San Diego, CA).

Cell culture and transfection. The SGC-7901 cells and COS-7 cells were cultured in medium supplied with $10 \%(\mathrm{v} / \mathrm{v})$ serum. The medium was changed every second day and the cells were subcultured at confluence. For transfection, the cells were subcultured the day before the process. The seeding amount of cells was adjusted to attain a density of $80-90 \%$ confluence on the day of transfection. Transfection was performed according to manufacturer's recommendations.

Preparation of Rhotekin-GST. The plasmid DNA encoding the RhoA-binding domain (RBD) of Rhotekin fused to glutathioneS-transferase (GST) was transfected into Escherichia coli. The bacteria were cultured at $37^{\circ} \mathrm{C}$ overnight and induced with isopropyl thiogalactoside (IPTG) at $30^{\circ} \mathrm{C}$ for $3 \mathrm{~h}$ to express protein. The bacteria cells were lysed with lysis buffer $[50 \mathrm{mM}$ Tris-Cl, pH 7.4, 1\% Nonidet P-40 (NP-40), $150 \mathrm{mM} \mathrm{NaCl}$, $5 \mathrm{mM} \mathrm{MgCl}{ }_{2}, 1 \mathrm{mM}$ dithiothreitol (DTT), $10 \mu \mathrm{g} / \mathrm{ml}$ of aprotinin, $10 \mu \mathrm{g} / \mathrm{ml}$ of leupeptin, and $1 \mathrm{mM}$ phenylmethylsulfonyl fluoride (PMSF)]. The lysate was centrifuged and the supernatant was incubated with glutathione beads at $4^{\circ} \mathrm{C}$ for at least $2 \mathrm{~h}$. The beads were washed several times with washing buffer [50 mM Tris-HCl, $\mathrm{pH} 7.4,0.5 \%$ Triton X-100; $150 \mathrm{mM} \mathrm{NaCl}, 5 \mathrm{mM} \mathrm{MgCl}_{2}$, and $1 \mathrm{mM}$ DTT]. After the final washing, the beads were suspended in washing buffer containing $10 \%$ (v/v) glycerol and kept at $-70^{\circ} \mathrm{C}$ until use.

RhoA-GTP pull-down assay. Rho activity was measured according to the method from Ren et al (16). Briefly, $3 \times 10^{6}$ cells were seeded on a $10-\mathrm{cm}$ dish. After the treatment, the cells were washed with Tris-buffered saline (TBS) and lysed with $400 \mu 1$ of lysis buffer containing $50 \mathrm{mM}$ Tris-Cl, $\mathrm{pH} 7.4$, $1 \%$ NP-40, $1 \%$ 3-[(3-cholamidopropy)dimethy-lammonio]-1propane sulfonate (CHAPS), $200 \mathrm{mM} \mathrm{NaCl}, 1 \mathrm{mM} \mathrm{MgCl}_{2}$, $10 \mu \mathrm{g} / \mathrm{ml}$ leupeptin, $10 \mu \mathrm{g} / \mathrm{ml}$ aprotinin, and $1 \mathrm{mM}$ PMSF. The cell lysate was centrifuged to eliminate the cell debris.
The supernatant $(10 \mu 1)$ was kept for loading control and the rest of the supernatant was incubated with GST-Rhotekinglutathione beads at $4^{\circ} \mathrm{C}$ for $45 \mathrm{~min}$, with continuous shaking. The beads were washed 3 times with a buffer [50 $\mathrm{mM}$ Tris-Cl, pH 7.4, 2\% NP-40, $200 \mathrm{mM} \mathrm{NaCl}$, and $10 \mathrm{mM}$ $\left.\mathrm{MgSO}_{4}\right]$. After the final washing, $20 \mu \mathrm{l}$ of $2 \mathrm{X}$ sodium dodecyl sulfate polyacrylamide gel electrophoresis (SDSPAGE) loading buffer was added to the beads, and the beads were boiled for $5 \mathrm{~min}$ to release proteins.

Western blotting. SDS-PAGE gels with different concentrations were cast according to the molecular size of target proteins. Sample proteins were accumulated with a voltage of $8 \mathrm{~V} / \mathrm{cm}$ and separated with a voltage of $15 \mathrm{~V} / \mathrm{cm}$ on the gel. After electrophoresis, the proteins on the gel were transferred to polyvinyl difluoride (PVDF) membrane, and the membrane was blocked with $3 \%(\mathrm{w} / \mathrm{v})$ of bovine serum albumin (BSA) in TBS-T for $1 \mathrm{~h}$ at room temperature (RT). The incubation with the primary antibody was overnight at $4{ }^{\circ} \mathrm{C}$, and the incubation with the secondary antibody was $50 \mathrm{~min}$ at RT, with 3 washes after each incubation. Electrochemiluminescence reagents were used to show the positive bands on the membrane. Briefly, solution A and solution B were mixed and added to the protein side of the membrane. The incubation was $5 \mathrm{~min}$ at RT. The exposure time of the first film was $15 \mathrm{sec}$. The exposure time of the second film was adjusted according to the extensity of the signal on the first film.

Luciferase reporter assay. The reporter gene plasmid pcDNASRE-Luc was co-transfected with the DNA construct of interest into cells grown in DMEM medium containing $10 \%$ (v/v) serum upon $60-80 \%$ cell confluence. $\beta$-galactosidase reporter vector was co-transfected as the control for transfection efficiency. After $24 \mathrm{~h}$, cells were stimulated with drugs for $4 \mathrm{~h}$. The Cells were washed twice with PBS and lysed in lysis buffer [25 mM glycylglycine ( $\mathrm{pH} 7.8), 1 \%$ Triton X-100, $15 \mathrm{mM} \mathrm{MgCl}_{2}, 1 \mathrm{mM}$ DTT] for $20 \mathrm{~min}$ on ice. The luciferase activity was measured in the presence of RAB buffer $[25 \mathrm{mM}$ glycine, $\mathrm{pH} 7.8,15 \mathrm{mM}$ potassium phosphate, $\mathrm{pH} 7.8,15 \mathrm{mM}$ $\mathrm{MgSO}_{4}, 4 \mathrm{mM}$ EGTA, $1 \mathrm{mM}$ dithiothreitol, and $1 \mathrm{mM}$ ATP] and 200nM D-luciferin. B-galactosidase activity was measured in the assay buffer [100 mM sodium phosphate, $\mathrm{pH} 7.3,1 \mathrm{mM} \mathrm{MgCl}_{2}, 50 \mathrm{mM}$ ß-mercaptoethanol, $0.665 \mathrm{mg} /$ $\mathrm{ml}$ o-nitrophenyl $\beta$-D-galactopyranoside]. The luciferase activities were calculated as the fold of increase after normalization by the $\beta$-galactosidase activity.

Immunofluorescence microscopy. The cells grown on cover slips were fixed with freshly prepared $2 \%(\mathrm{w} / \mathrm{v})$ paraformaldehyde in PBS for $15 \mathrm{~min}$, permeabilized with $0.3 \%$ Triton X-100 in PBS for $10 \mathrm{~min}$, and washed in PBS for $10 \mathrm{~min}$. Cells were then stained with $50 \mathrm{ng} / \mathrm{ml}$ rhodamineconjugated phalloidin (to visualize filamentous actin) for $1 \mathrm{~h}$ at room temperature, washed with $0.1 \%$ Triton X-100 in PBS three times for $5 \mathrm{~min}$ each. The morphologic changes of the cells were viewed under a fluorescent microscope.

Stably transfected SGC-7901 cells. SGC-7901 cells were co-transfected with $300 \mathrm{ng}$ pcDNA-HT31wt and $300 \mathrm{ng}$ pcDNA3.1-GFP using lipofectamine 2000 (Invitrogen). 

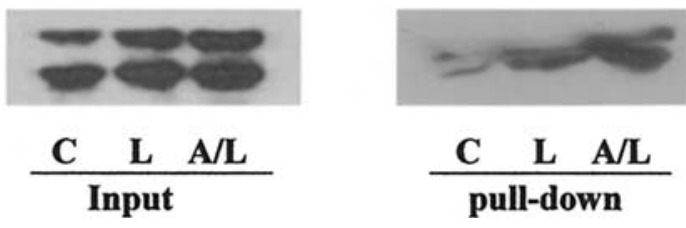

Figure 1. RhoA activity of transfected COS-7 cells treated with CPT-cAMP followed by LPA. The cells were co-transfected with HT31 wild-type plasmid and RhoA wild-type plasmid containing EE tag. RhoA-GTP was measured by pull down assay. Lane C, control; Lane L, cells stimulated with LPA alone for $10 \mathrm{~min}$; Lane A/L, cells treated with $100 \mu \mathrm{M}$ CPT-cAMP for $30 \mathrm{~min}$ followed by $1 \mu \mathrm{M}$ LPA for $10 \mathrm{~min}$. There were two bands in each lane. The upper bands represent exogenous RhoA with EE tag. The lower bands represent endogenous RhoA. A representative result of three experiments is shown.

Stably transfected cells were selected with G418 (500 ng/ml) and two clones of SGC-7901/HT31wt were eventually expanded. The expression of HT31wt protein by the cells was verified by electrophoresis.

Scrape motility assay. SGC-7901/HT31wt cells were grown to confluence on 24-well plate. The cell monolayer was mechanically scarred with a sterile rubber scraper, and the plate was placed in the incubator, which maintained the temperature at $37^{\circ} \mathrm{C}$ and an atmosphere containing $5 \%(\mathrm{v} / \mathrm{v})$ $\mathrm{CO}_{2}$. Cells were visualized by a Nikon reverse microscope with phase contrast optics lens. Drugs were added directly to the culture media at the time of scraping. The media was not changed throughout the recording time.

Anchorage-independent growth assay (17). The base agar was prepared by mixing equal volumes of $1 \%(\mathrm{w} / \mathrm{v})$ agar and $2 \mathrm{X}$ $\mathrm{DMEM}+20 \%(\mathrm{v} / \mathrm{v})$ serum. The top agar was prepared by mixing equal volumes of $0.7 \%(\mathrm{w} / \mathrm{v})$ agarose (DNA grade) and 2 X DMEM $+20 \%(\mathrm{v} / \mathrm{v})$ serum, with 5000 cells/ per 30-mm dish suspended in the agar. The dish was incubated at $37^{\circ} \mathrm{C}, 5 \%(\mathrm{v} / \mathrm{v}) \mathrm{CO}_{2}$ in a humidified incubator. The cell proliferation was observed and recorded under a reverse microscope. From the second day on, cell clusters could be seen, which represented the early stage of the anchorage-independent growth. Random fields were selected and both the number of cell clusters containing several cells and the number of total cells seeded in the fields were counted. Ratio of cluster formation (the number of cell cluster divided by the number of total cells seeded) was calculated to represent the early proliferation of the cells.

Statistics. The data are expressed as means \pm standard deviation (SD). Statistical significance was tested with Student's t-test, and $\mathrm{P}<0.05$ was accepted as a significant difference.

\section{Results}

HT31 abolished the inhibitory effect of CPT-cAMP on RhoA activation. The result of RhoA-GTP pull down assay showed that RhoA activity increased in SGC-7901 cells treated with LPA and pre-treating cells with membrane-permeable cAMP analog CPT-cAMP inhibited RhoA activation induced by LPA (similar to published result, not shown). But the effect
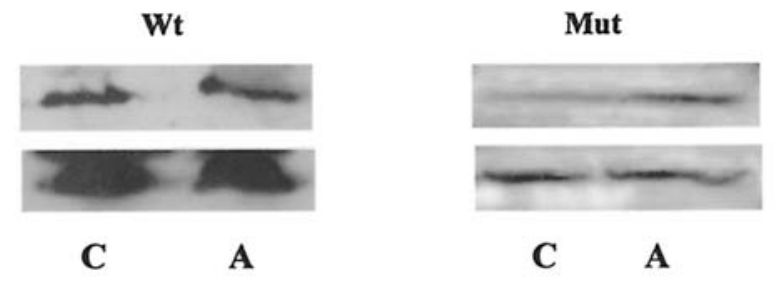

Figure 2. RhoA phosphorylation at serine ${ }^{188}$ induced by CPT-cAMP in Cos-7 cells transfected with HT31 wild/mutant type plasmid. After transfected with HT31wt/mut DNA constructs, the cells were treated with CPT-cAMP, and the cell lysate was detected by Western blotting with antibodies against RhoA phosphorylated at serine ${ }^{188}$ and normal RhoA. The left panels show RhoA phosphorylation in cells transfected with HT31 wild-type construct. The right panels show RhoA phosphorylation in cells transfected with HT31 mutant type construct. The upper panels are the result of Western blotting with antibody against RhoA phosphorylated at serine ${ }^{188}$. The lower panels are the result of Western blotting with antibody against normal RhoA. Lane C, control cells; Lane A, cells treated with $100 \mu \mathrm{M}$ CPT-cAMP for $30 \mathrm{~min}$. A representative result of three experiments is shown.

of LPA could not be inhibited by CPT-cAMP in COS-7 cells co-transfected with pcDNA-HT31wt and pcDNA-EE-RhoAwt. Both exogenous and endogenous GTP-RhoA was increased upon LPA stimulation with CPT-cAMP pre-treatment (Fig. 1).

HT31 blocked RhoA serine ${ }^{188}$ phosphorylation caused by $C P T$-cAMP. PKA is known to inhibit RhoA activation by phosphorylating serine ${ }^{188}$ of RhoA. To determine the phosphorylation of RhoA induced by CPT-cAMP, an antibody against RhoA phosphorylated at serine ${ }^{188}$ was used in Western blotting. The result showed that there was hardly any phosphorylation of RhoA proteins in control cells. However, in cells treated with CPT-cAMP, the phosphorylation level increased notably in a dosage-dependent manner (similar to published result, not shown). To determine whether the destruction of PKA anchorage would affect the phosphrylation of RhoA caused by CPT-cAMP or not, we transfected the SGC-7901 cells with pcDNA-HT31wt/mut. In cells transfected with pcDNA-HT31wt, the phosphorylation level of the cells treated by CPT-cAMP had no significant difference from that of the control cells. In contrast, in cells transfected with pcDNA-HT31mut, the phosphorylation increased obviously after CPT-cAMP treatment (Fig. 2).

Effect of HT31 on LPA and CPT-cAMP induced SREdependent transcription. Reporter gene assay showed that LPA stimulation caused about 8-fold increase of SREdependent transcription in SGC-7901. Treating cells with both serum and LPA significantly increased the transcription activity while treating the cells with CPT-cAMP had little effect on basal transcription of the SRE-dependent reporter gene (Fig. 3A). In cells transfected with pcDNA-HT31wt, LPA-stimulated SRE-dependent transcription could not be inhibited by pre-treating cells with CPT-cAMP (Fig. 3B). Whereas the transcription decreased significantly in cells transfected with pcDNA-HT31mut after the same treatment (Fig. 3C).

Action of CPT-cAMP/LPA on LPA-induced stress fiber formation in SGC-7901 cells transfected with pcDNA HT31wt/ $m u t$. To detect the effect of destroying the PKA anchorage on 
the LPA-induced formation of stress fiber, we transfected the cells with pcDNA-HT31wt/mut and stained F-actin with rhodamine-conjugated phalloidin. In the cells transfected with pcDNA-HT31 wt, LPA greatly increased the formation of stress fibers and CPT-cAMP pre-treatment could not inhibit LPA-induced stress fiber formation (Fig. 4A). In cells transfected with pcDNA-HT31mut, the formation of stress fibers stimulated by LPA could be inhibited by CPT-cAMP (Fig. 4B).

The motility and anchorage-independent growth in cells highly expressing HT31. To investigate whether PKA still inhibited LPA-induced biological activities such as cell motility and anchorage-independent growth after its anchorage was destroyed by HT31, we co-transfected SGC-7901 cells with pcDNA-HT31 and pGFP, and selected clones which stably expressed both HT31 peptide and GFP. The expression of both HT31 and GFP was confirmed by electrophoresis. The stably transfected cells were used in the following experiments.

In scrape motility assay, a few untransfected SGC-7901 cells migrated over the borderline $24 \mathrm{~h}$ after scraping. Treating cells with LPA promoted the migration of the cells, and pre-treating cells with CPT-cAMP inhibited the LPAinduced cell migration (similar to published result, not shown). In the cells expressing both HT31 peptide and GFP, however, the inhibitory action of CPT-cAMP did not exist. The cells treated with CPT-cAMP and then LPA migrated similarly to the cells treated with LPA only (Fig. 5A).

The short time effect of LPA on growth in soft agar was observed and there were more clusters in cells treated with LPA than in control cells and CPT-cAMP prevented the increase. The result of this experiment showed that in the cells stably expressing HT31 peptide, the growth of the cells treated with CPT-cAMP and LPA had no significant difference from that of the cells treated with LPA alone (Fig. 5B).

\section{Discussion}

Previous investigation in our laboratory provided evidence that cAMP/PKA signaling inhibited RhoA activation through phosphorylating RhoA on serine ${ }^{188}$ and antagonized RhoA functions in regulating stress fiber formation, morphology change, motility and anchorage-independent growth of cancer cells (18). In order to explore the mechanism further, this experiment was aimed to investigate the role of PKA anchorage in inhibiting RhoA activity by the kinase. We demonstrated that AKAP-mediated PKA anchorage was necessary for the kinase to phosphorylate RhoA and to regulate RhoA activation and RhoA-related cellular activities. This is the first study to examine the effect of blocking PKA anchorage on the crosstalk between PKA- and RhoA-mediated signal transductions.

The ability of cAMP/PKA to prevent the activation of RhoA has been proved in different cell types. Phosphorylating RhoA on $\operatorname{ser}^{188}$ by PKA inhibits RhoA activity in vitro and in vivo, through promoting formation of RhoA-RhoGDI complexes and enhancing the ability of RhoGDI to extract RhoA from membranes $(19,20)$. Our previous results showed that $\mathrm{CAMP} / \mathrm{PKA}$ prevented the activation of RhoA in human

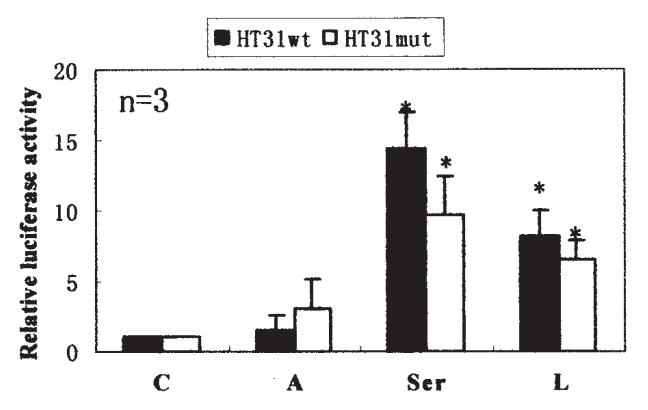

$\mathbf{A}$

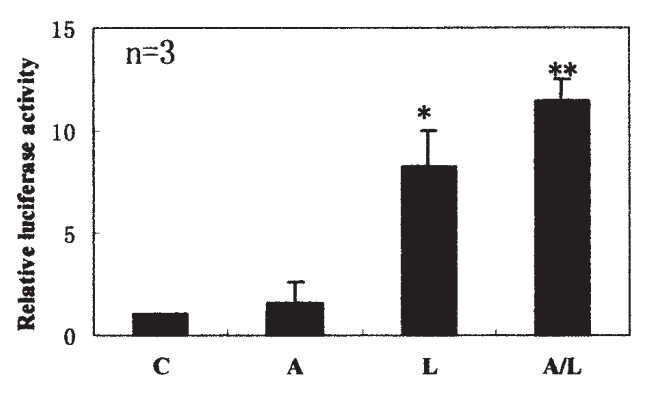

B

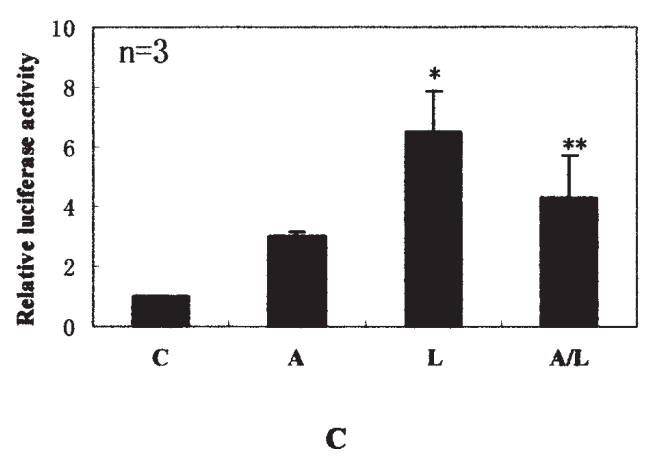

Figure 3. HT31 abolished PKA inhibition on LPA-induced SRE-dependent transcription in SGC-7901 cells. The cells were transfected with pSRE-luc and $\mathrm{p} ß \mathrm{Gal}$ and co-transfected with pHT31-mut, or pHT31-wt, or empty vector. Firefly luciferase activities were normalized to $\beta$-galactosidase activities in each sample, and the relative luciferase activity measured in untreated cells (1st column) was assigned the value of 1. (A) Cells transfected with pHT31-mut, or pHT31-wt were treated with CPT-cAMP, serum, or LPA respectively for $4 \mathrm{~h}$. (B) Cells transfected with pHT31-wt were incubated for $45 \mathrm{~min}$ in the absence or presence of CPT-cAMP and stimulated with LPA for $4 \mathrm{~h}$. (C) Cells transfected with pHT31-mut following the same treatment as in (B). C: control; Ser: treated with $20 \%$ serum; A: treated with $250 \mu \mathrm{M}$ CPT-cAMP; L: treated with $1 \mu \mathrm{M} \mathrm{LPA}$; $\mathrm{A} / \mathrm{L}$ : treated with $250 \mu \mathrm{M}$ CPT-cAMP $+1 \mu \mathrm{M} \mathrm{LPA} .{ }^{*} \mathrm{p}<0.05$ compared with control $;{ }^{* *} \mathrm{p}<0.05$ compared with cells treated with LPA.

gastric cancer cell line SGC-7901. In this experiment, we showed that in cells transfected with PKA anchorage inhibitory peptide HT31, the pre-treatment with CPT-cAMP could not inhibit the RhoA activation caused by LPA. This suggested that the expression of the peptide in the cells prevented the inhibition of RhoA activity by PKA. To confirm if the prevention was caused by the reduction of PKA phosphorylating on RhoA or not, we detected the phosphorylation of RhoA by PKA in presence/absent of HT31. The result showed that the level of phosphorylation was lower in cells 
A

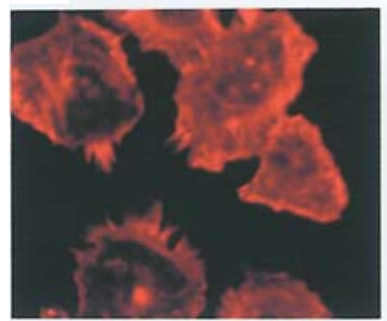

a

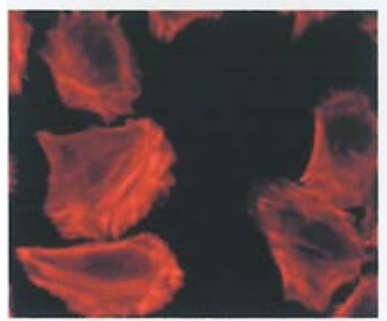

C

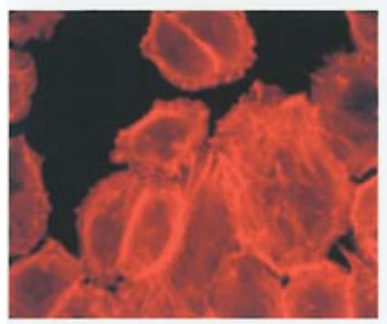

e

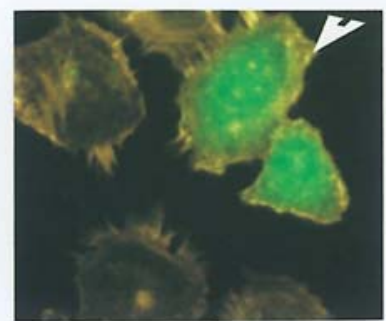

b

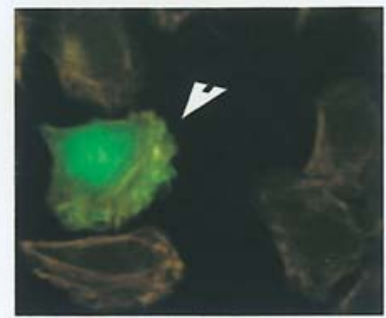

d

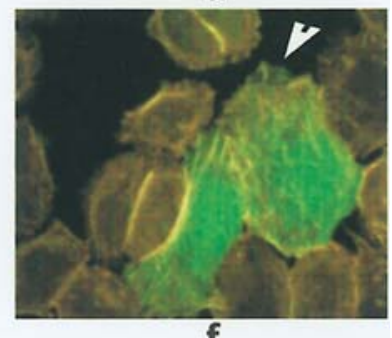

B

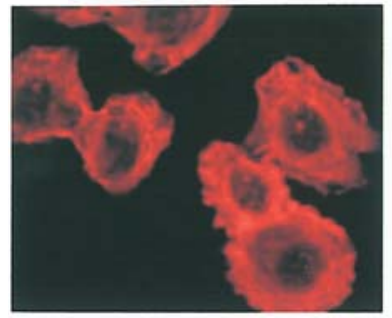

a

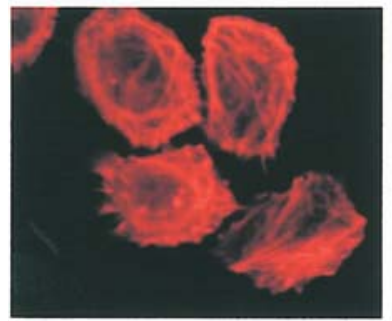

C

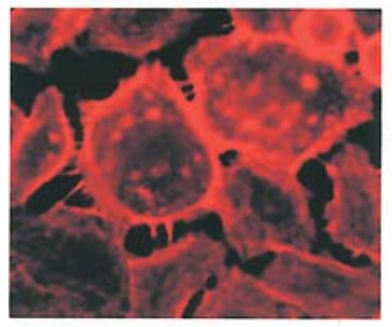

e

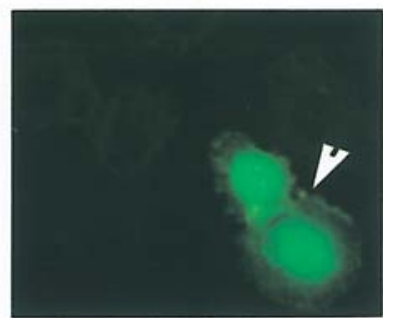

b

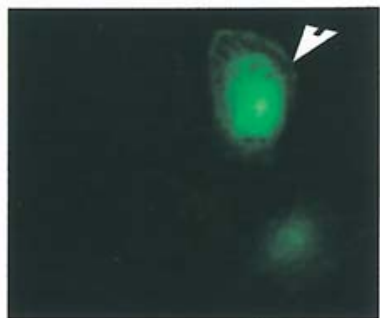

d

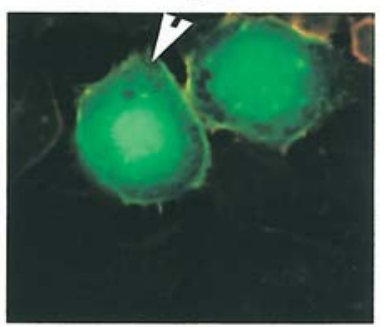

f

Figure 4. (A) Effect of CPT-cAMP on LPA-induced stress fiber formation in SGC-7901 cells transfected with pHT31 wt. The cells were transfected with pHT31 wt and pGFP and treated with LPA or CPT-cAMP and then LPA and were processed for fluorescent microscope observation as described in materials and methods. (a) and (b), cells without stimulation visualized under green and blue fluorescent, respectively; (c) and (d), cells treated with $1 \mu \mathrm{M}$ LPA for $15 \mathrm{~min}$; (e) and (f), cells treated with $250 \mu \mathrm{M}$ cAMP for 30 min and then with $1 \mu \mathrm{M}$ LPA for 15 min. Original magnification: 4400 for fluorescence microscopy photos. Arrows indicated the cells successfully transfected. (B) Effect of CPT-cAMP on LPA-induced stress fiber formation in SGC-7901 cells transfected with pHT31 mut. The cells were transfected with pHT31mut and pGFP and treated with LPA or CPT-cAMP and then LPA and were processed for fluorescent microscope observation as described in Materials and methods. (a) and (b), cells without stimulation visualized under green and blue fluorescent, respectively; (c) and (d), cells treated with $1 \mu \mathrm{M}$ LPA for $15 \mathrm{~min}$; (e) and (f), cells treated with $250 \mu \mathrm{M}$ cAMP for 30 min and then with $1 \mu \mathrm{M}$ LPA for 15 min. Original magnification: $\mathrm{x} 400$ for fluorescence microscopy photos. Arrows indicated the cells successfully transfected.

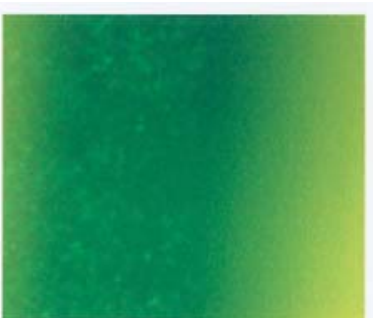

a

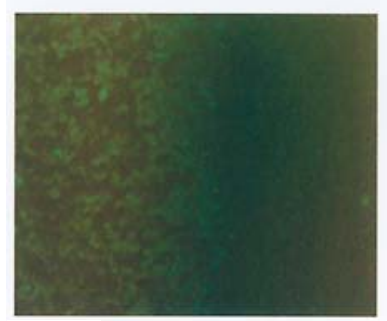

A

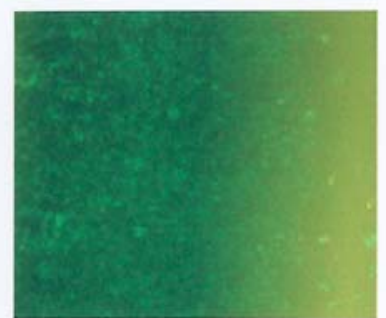

b

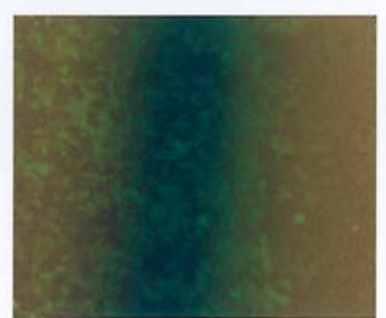

d

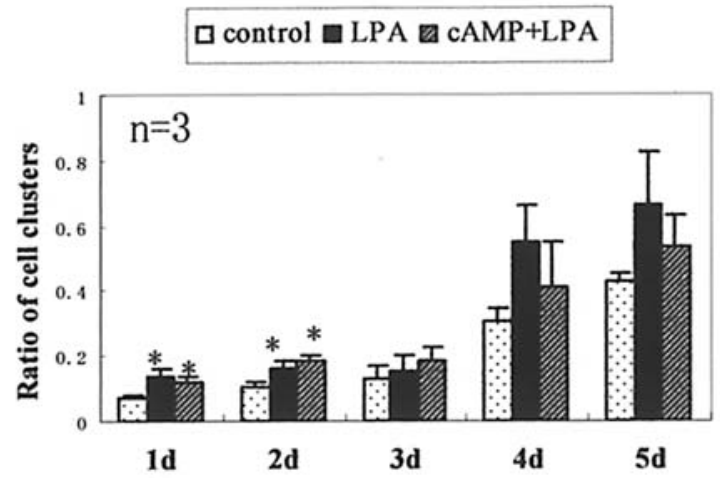

B

Figure 5. Migration and anchorage-independent growth of stably transfected SGC-7901 cells expressing HT31wt. (A) In scrape motility assay, treating the stably transfected SGC-7901 cells with LPA caused the migration into the scraped area and CPT-cAMP could not inhibit the migration. a: control; b: cells treated with $1 \mu \mathrm{M} \mathrm{LPA}$; c: cells treated with $250 \mu \mathrm{M}$ CPT-cAMP; d: cells treated with $250 \mu \mathrm{M}$ CPT-cAMP $+1 \mu \mathrm{M}$ LPA. (B) The stably transfected SGC-7901 cells were cultured in soft agar. Ratio of cell clusters (number of clusters divided by number of total seeded cells in the randomly selected fields) was calculated to represent the early proliferation of the cells. The results are the means $\pm \mathrm{SD}$ of three independent experiments. ${ }^{*} \mathrm{P}<0.05$ compared with control. 
transfected with wild-type HT31 than that in cells transfected with mutant HT31. This meant that the loss of PKA inhibition on RhoA activation after the disruption of PKA anchorage was due to the decrease of phosphorylation of RhoA caused by PKA.

Subcellular localization of PKA is mainly due to anchoring of the R subunits by AKAPs, which is understood to enhance the efficiency and specificity of the signaling events (21). Sequestering PKA to different subcellular locations would allow the kinase pool closest to its substrates and then phosphorylate nearby protein substrates. For example, several AKAPs may function to sequester PKA near its major targets in the heart $(22,23)$. So the relative localization of PKA-AKAP complex and RhoA is very important for PKA phosphorylation of RhoA. In addition, several AKAPs are also able to form multivalent signal transduction complexes by interaction with phosphatases as well as other kinases and proteins. Moreover, it has been reported that HT31 protein can link with RhoA in vivo (24). The details of the physical relation among PKA, RhoA and HT31 needs further investigation.

RhoA is highly expressed in cancer cells and tightly related with the biological events of cancer cells such as gene transcription, motility-related morphological change, anchorage-independent growth and invasiveness and metastasis. Activation of RhoA can stimulate serum response element (SRE) dependent gene transcription through activating serum response factor $(\mathrm{SRF})(25,26)$. To date, no result shows that cAMP solely affects the SRE-dependent transcription. However, the SRE-dependent transcription induced by a growth factor can be inhibited by cAMP in breast cancer cells (27) and cAMP can inhibit the RhoA-63L induced SREdependent transcription (26). Our data not only showed that cAMP inhibited the SRE-dependent transcription stimulated by LPA but also that PKA inhibition on RhoA-mediated SRE-dependent transcription disappeared after the disruption of AKAP-mediated PKA anchorage.

RhoA is a key protein in the process of assembling stress fibers and participates in the formation of focal adhesions following cell-matrix interactions (28). Research data also indicate that RhoA and cAMP have antagonistic roles in regulating cellular morphology and suggest that cAMPmediated down-regulation of RhoA binding to its effector ROCK may be involved in this antagonism (8). Accumulating evidence shows that AKAP mediated organization of kinases and phosphatases is particularly important for the transduction of signals to the cytoskeleton (29). In this experiment, we focus on the role of AKAPs in the crosstalk of PKA- and RhoA-meidated signal transduction pathways in stress fiber formation. Our result revealed that PKA-mediated downregulation of RhoA function in stress fiber formation was also achieved by AKAP-mediated anchoring of PKA.

The important role of RhoA has been highlighted in gastric cancer invasiveness suggesting that it may serve as a target in future strategies at blocking invasion by these tumors (30-32). Previous result of our laboratory presented that PKA activation inhibited RhoA function in invasionrelated biological activities such as cell migration and anchorage-independent growth. In this study, we investigated similar activation induced by RhoA after disruption of anchorage of PKA. The results show that in presence of
HT31, the inhibition disappeared of PKA on RhoA related migration and anchorage-independent growth.

In conclusion, AKAP-mediated PKA anchorage is necessary for PKA to inhibit RhoA activation and RhoArelated biological activities induced by LPA in cancer cells. These effects occur likely through reduction of phosphorylation of RhoA caused by PKA.

\section{Acknowledgements}

This study was supported by the National Natural Science Foundation of China Grants No. 30340036 and No. 30470891 , and Grant of Zhenjiang Key Institute of Clinical Laboratory Medicine No. SH2006066. We thank Professor R.B. Pilz, University of California, CA, USA for the kind gifts of DNA constructs.

\section{References}

1. Takai Y, Sasaki T and Matozaki T: Small GTP-binding proteins: Review. Physiol Rev 81: 153-208, 2001.

2. Sahai E and Marshall CJ: RHO-GTPases and cancer: Review. Nat Rev Cancer 2: 133-142, 2002.

3. Kjoller L and Hall A: Signaling to Rho GTPases: Review. Exp Cell Res 25: 166-179, 1999.

4. Prendergast GC, Khosravi-Far R, Solski PA, Kurzawa H, Lebowitz PF and Der C: Critical role of Rho in cell transformation by oncogenic Ras. Oncogene 10: 2289-2296, 1995.

5. Sahai E, Olson MF and Marshall C: Cross-talk between Ras and Rho signalling pathways in transformation favours proliferation and increased motility. EMBO J 20: 755-766, 2001.

6. Lang P, Gesbert F, Delespine-Carmagnat M, Stancou R, Pouchelet $\mathrm{M}$ and Bertoglia J: Protein kinase A phosphorylation of RhoA mediates the morphological and functional effects of cyclic AMP in cytotoxic lymphocytes. EMBO J 15: 510-519, 1996.

7. Sawada N, Itoh H, Yamashita J, Doi K, Inoue M, Masatsgugu K, Fukunaga Y, Sakaguchi S, Sone M, Yamahara K, Yurugi T and Nakao K: cGMP-dependent protein kinase phosphorylates and inactivates RhoA. Biochem Biophys Res Commun 280: 798-805, 2001.

8. Dong JM and Leung T: cAMP-induced morphological changes are counteracted by the activated RhoA small GTPase and the Rho Kinase ROKa. J Biol Chem 273: 22554-22562, 1998.

9. O'Connor KL, Nguyen BK and Mercurio AM: RhoA function in lamellae formation and migration is regulated by the alpha6beta4 integrin and cAMP metabolism. J Cell Biol 148: 253-258, 2000.

10. Beebe SJ and Corbin JD: The Enzymes: Control by phosphorylation. Academic, New York, NY, pp43-111, 1986.

11. Dell'Acqua ML and Scott JD: Protein kinase A anchoring: Review. J Biol Chem 272: 12881-12884, 1997.

12. Carr DW, Renata ES, Fraser IDC, Bishop SM, Scott TS, Brennan RG and Scott JD: Interaction of the regulatory subunit of cAMP-dependent protein kinase with RII-anchoring proteins occurs through an amphipathic helix binding motif. J Biol Chem 266: 14188-14192, 1991.

13. Carr DW, Hausken ZE, Fraser ID, Stofko-Hahn RE and Scott JD: Association of the type II cAMP-dependent protein kinase with a human thyroid RII-anchoring protein. Cloning and characterization of the RII-binding domain. J Biol Chem 267: 13376-13382, 1992.

14. Fink MA, Zakhary DR, Mackey JA, Apperson-Hansen C, Desnoyer RW, Damron DS and Bond M: AKAP-mediated targeting of protein kinase A regulates contractility in cardiac myocytes. Circ Res 88: 291-297, 2001.

15. Vijayaraghavan S, Goueli SA, Davey MP and Carr DW: Protein kinase A - anchoring inhibitor peptides arrest mammalian sperm motility. J Biol Chem 272: 4747-4752, 1997.

16. Ren XD, Kiosses WB and Schwartz MA: Regulation of the small GTP binding protein Rho by cell adhesion and the cytoskeleton. EMBO J 18: 578-585, 1999.

17. Horowitz D and King AG. Colorimetric determination of inhibition of hematopoietic progenitor cells in soft agar. Immunol Methods 244: 49-58, 2000. 
18. Chen Y, Wang Y, Yu H, Wand F and Xu W: The cross talk between protein kinase A- and RhoA-mediated signaling in cancer cells. Exp Biol Med 230: 731-741, 2005.

19. Qiao J, Huang F and Lum H: PKA inhibits RhoA activation: a protection mechanism against endothelial barrier dysfunction. Am J Physiol Lung Cell Mol Physiol 284: L972-L980, 2003.

20. Ellerbroek SM, Wennerberg K and Burridge K: Serine phosphorylation negatively regulates RhoA in vivo. J Bio Chem 278: 19023-19031, 2003.

21. Colledge M and Scott JD: AKAPs: from structure to function. Trends Cell Biol 9: 216-221, 1999.

22. Ruehr ML, Russell MA and Bond M: A-kinase anchoring protein targeting of protein kinase A in the heart. J Mol Cell Cardiol 37: 653-665, 2004.

23. Gao T, Yatani A, Dell'Acqua M, Sako H, Green S, Dascal N, Scott J and Hosey M: cAMP-dependent regulation of cardiac Ltype $\mathrm{Ca}$ channels requires membrane targeting of PKA and phosphorylation of channel subunits. Neuron 19: 185-196, 1997.

24. Klussmann E, Edemir B, Pepperle B, Klauschenz VHE, Tamma G, Hundsrucker C, Maric K and Rosenthal W: HT31: the first protein kinase $\mathrm{A}$ anchoring protein to integrate protein kinase A and Rho signaling. FEBS Lett 507: 264-268, 2001.

25. Chai J and Tarnawski AS: Serum response factor: discovery, biochemistry, biological roles and implications for tissue injury healing. Review. J Physiol Pharmacol 53: 147-157, 2002.
26. Gudi T, Chen JC, Casteel DE, Seasholtz TM, Boss GR and Pilz RB: cGMP-dependent protein kinase inhibits serumresponse element-dependent transcription by inhibiting Rho activation and functions. J Bio Chem 277: 37382-37393, 2002.

27. Lowe WL, Fu R and Banko M: Growth factor-induced transcription via the serum response element is inhibited by cyclic adenosine 3',5'-monophosphate in MCF-7 breast cancer cells. Endocrinology 138: 2219-2226, 1997.

28. Ridley AJ and Hall A: The small GTP binding protein rho regulates the assembly of focal adhesions and actin stress fibers in response to growth factors. Cell 70: 389-399, 1992.

29. Diviani D and Scott JD: AKAP signaling complexes at the cytoskeleton. Review. J Cell Sci 114: 1431-1437, 2001.

30. Ridley AJ: RhoGTPases and cell migration. J Cell Sci 114: 2713-2722, 2001.

31. Michiels F and Collard JG: Rho-like GTPases: their role in cell adhesion and invasion. Biochem Soc Symp 65: 125-146, 1999.

32. Stahle M, Veit C, Bachfischer U, Schierling K, Skripczynski B, Hall A, Gierschik P and Giehl K: Mechanisms in LPA-induced tumor cell migration: critical role of phosphorylated ERK. J Cell Sci 116: 3835-3846, 2003 